\title{
Quantifying the Structural Dynamics of Pharmaceuticals in the Glassy State
}

\author{
Z. Wojnarowska, ${ }^{* \dagger}$ C. M. Roland, ${ }^{\dagger}$ K. Kolodziejczyk, ${ }^{\dagger}$ A. Swiety-Pospiech, ${ }^{\dagger}$ K. Grzybowska, ${ }^{\dagger}$ \\ and M. Paluch ${ }^{\dagger}$ \\ ${ }^{\dagger}$ Institute of Physics, Silesian University, ul. Uniwersytecka 4, 40-007 Katowice, Poland \\ ${ }^{\ddagger}$ Chemistry Division, Naval Research Laboratory, Code 6120, Washington, DC 20375-5342, United States
}

ABSTRACT: Structural dynamics in the glassy state of two protic ionic liquids, carvedilol phosphate and procaine hydrochloride, were characterized from analysis of changes in the conductivity relaxation times during physical aging. The obtained relaxation times, having a magnitude exceeding feasible experimental time scales and thus not directly measurable, are consistent with published data from a method that relies on the presence of a secondary relaxation. We also observe a narrowing of the relaxation dispersion, specific to higher frequencies, that is a consequence of the heterogeneous dynamics of deeply super-

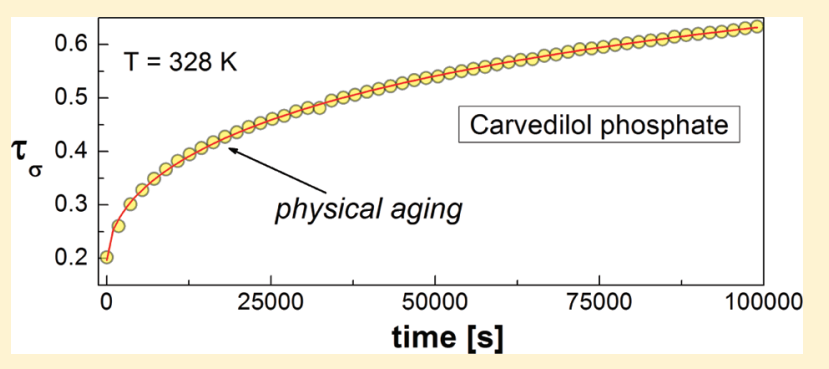
cooled materials.

SECTION: Glasses, Colloids, Polymers, and Soft Matter

$\mathrm{T}$ he irony of the $\sim 100$ years of research on the glass transition is that so little of it is concerned with glasses per se. This is the case even though the vitreous state is associated with distinct properties that may help solve the glass transition problem, properties such as nonergodicity, an Arrhenius temperature-dependence for the primary relaxation time, and invariance to thermodynamic conditions of both the shape of the relaxation function and the dynamic correlation volume. The limited experimental data on glassy dynamics obviously results from the inability of typical dynamic spectroscopy to measure structural relaxation occurring over prolonged time periods. Approaches to circumvent this difficulty include addition to the sample of chromophores, whose motion can be monitored without the need for motion of the host molecules, ${ }^{1,2}$ and measurement of secondary relaxations, from which inferences are drawn concerning the structural dynamics. $^{3-6}$

The principle material studied herein is carvedilol phosphate (CP, structure in Figure 1), which is comprised of a racemic mixture of two enantiomers and usually exists as the hemihydrate. The substance finds application as an oral medicine, used in the treatment of hypertension and certain heart abnormalities. Our interest is the behavior of glassy $\mathrm{CP}$ because the glassy state of pharmaceuticals is an attractive means to control the solubility and bioavailability of drugs. However, glasses are metastable, hence the possibility of crystallization or other physical changes that may degrade biological function. The tendency for such changes is related to the molecular mobility of the glass, which makes pharmaceuticals an important class of materials for studies of glassy dynamics. $^{7-16}$ In this work, we describe a new approach, in which the change in the diffusivity of ions due to physical aging

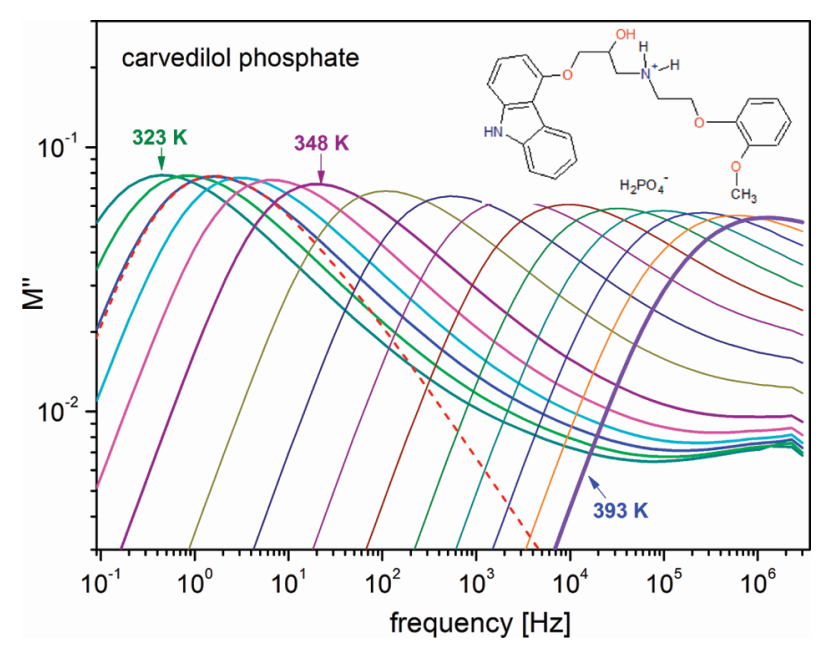

Figure 1. Peak in the dielectric loss modulus due to conductivity relaxation at various temperatures in the liquid and glassy states (the interval between each spectrum is $5 \mathrm{~K}$ ). The dashed line is the Kohlrausch function with $\beta_{\mathrm{KWW}}=0.53$ fitted to the loss peak at $333 \mathrm{~K}$. The chemical structure of $\mathrm{CP}$ is shown.

is used to define a time constant that characterizes structural relaxation of the glass. The idea underlying this method is that physical aging is governed by the time constant measured in suitable dielectric experiments (e.g., nonlinear spectroscopy $^{17-20}$ and hole-burning experiments $\left.{ }^{21}\right)$; that is, subensembles

Received: March 20, 2012

Accepted: April 23, 2012

Published: April 23, 2012 
within the glass proceed toward equilibrium at a rate reflected in the rate of their local dynamics. We verify the method by applying it to ion diffusion data in another drug, procaine hydrochloride. In addition to extracting relaxation times for the glassy state, we also observe in $\mathrm{CP}$ an asymmetric narrowing of the dispersion of the conductivity relaxation. Such a result implies a reduction in the distribution of relaxation times and inferentially of the structural heterogeneity of the glass. ${ }^{22}$ The behavior is consistent with the high-frequency modes having shorter relaxation times and in turn a faster response to the volume changes induced by physical aging. ${ }^{23}$

The dc conductivity relaxation spectra due to diffusion of the dihydrogen phosphate anions $\left(\mathrm{H}_{2} \mathrm{PO}_{4}^{-}\right)$in $\mathrm{CP}$ were measured with a Novocontrol Alpha analyzer. To probe structural relaxation directly, stochastic temperature-modulated differential scanning calorimetry (TMDSC) was carried out using a Mettler-Toledo TOPEM. The carvedilol phosphate with a chemical purity greater than $99.5 \%$ was obtained from Polpharma Pharmaceutical Works (Poland) and used as received.

Figure 1 displays the loss component of the complex electric modulus, $M^{\prime \prime}$, measured over a $70^{\circ}$ range encompassing the glass transition temperature (calorimetric $T_{\mathrm{g}}=351 \mathrm{~K}$ evaluated from the temperature dependence of the quasi-static heat capacity $\left.c_{\mathrm{p} 0}\right)$.

The peak due to ion motion completely masks the primary $\alpha$-relaxation even above $T_{\mathrm{g}}$ because of the high ion concentration ( $\mathrm{CP}$ is a protic ionic liquid ${ }^{24,25}$ ). As the temperature decreases, the dispersion moves to lower frequencies; however, the effect of temperature is markedly reduced below $T_{\mathrm{g}}$. This is evident in the relaxation map in Figure 2, showing the $\mathrm{dc}$ conductivity relaxation time, $\tau_{\sigma}$, defined as the inverse of the frequency of the $M^{\prime \prime}$ peak.

Ion diffusion continues below $T_{\mathrm{g}}$, but the cessation of rotational motion of the $\mathrm{CP}$ molecule inhibits the migration, increasing $\tau_{\sigma}$ and reducing its sensitivity to $T$. Such decoupling of ion translation and host molecule reorientation has been reported before in other glassy materials. ${ }^{26-28}$ For comparison, included in Figure 2 are the $\alpha$-relaxation times determined by TMDSC from the temperature dependence of the real part of the complex heat capacity, $c_{\mathrm{p}}{ }^{\prime}(T)$, measured at different frequencies (see the inset to Figure 2). Although the calorimetric relaxation times do not precisely correspond to the values from dielectric spectroscopy, these $\tau_{\alpha}$ are certainly much longer, by $\sim 3$ orders of magnitude, than the ion relaxation times.

$\tau_{\sigma}(T)$ becomes Arrhenius below $T_{\mathrm{g}}$, with an activation energy equal to $115 \pm 2 \mathrm{~kJ} / \mathrm{mol}$. Because the glass is metastable, the density increases over time as the material slowly equilibrates (physical aging). This imparts a time dependence to the properties, and slowing of ion diffusion is evident in Figure 3 in the shift of the $M^{\prime \prime}$ peak toward lower frequencies. However, the effect of physical aging is not limited to changing the mean relaxation time; there is a systematic narrowing of the dispersion on the high-frequency side, as seen in the inset to Figure 3.

We quantify the change in the conductivity relaxation times by fitting the data for three temperatures, all below $T_{g}$, to an empirical equation

$$
\tau_{\sigma}=A \exp \left(-\left[t / \tau_{\text {age }}\right]^{\beta}\right)+\tau_{\sigma, \infty}
$$

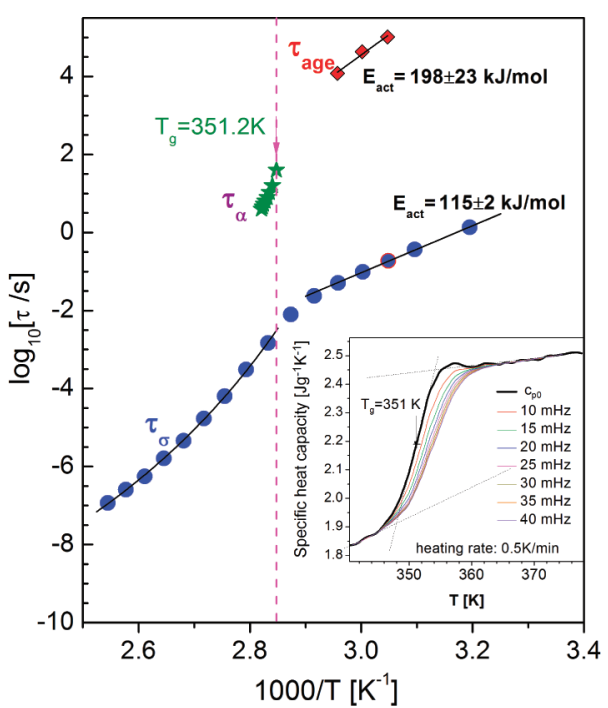

Figure 2. Conductivity relaxation times for $\mathrm{CP}$ (circles), showing the weaker $T$ dependence below $T_{\mathrm{g}}$, and the structural relaxation times measured by TMDSC (stars) and extracted from the change in $\tau_{\sigma}$ during physical aging (diamonds). The lines are Arrhenius fits to the data below $T_{\mathrm{g}}$ (activation energies as indicated) and to the Vogel equation for $\tau_{\sigma}$ of the liquid, $\log \left(\tau_{\sigma} / \mathrm{s}\right)=-14.82+910.4 /(T-277.2)$. $T_{g}$, determined as the temperature at the midpoint of the step change in $c_{\mathrm{p} 0}(T)$, is indicated by the vertical dashed line. The inset shows the temperature dependences of the real part of complex specific heat capacity $c_{\mathrm{p}}{ }^{\prime}(T)$ measured at different frequencies, along with the quasistatic specific heat capacity $c_{\mathrm{p} 0}(T)$.

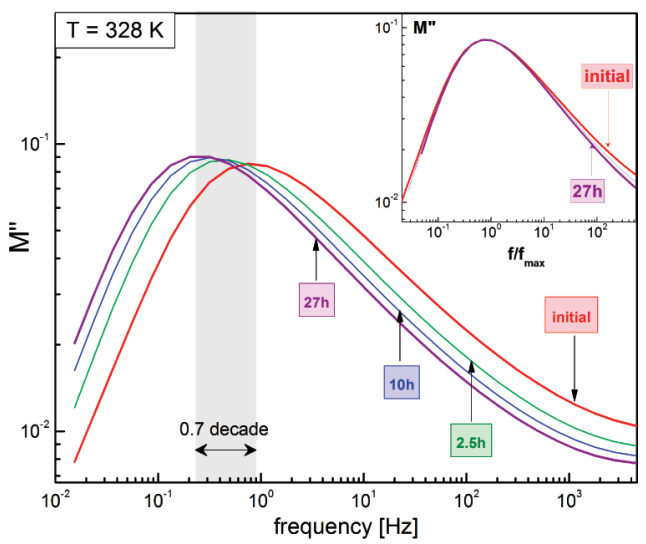

Figure 3. Shift of the conductivity peak during physical aging at a temperature $26 \mathrm{~K}$ below $T_{\mathrm{g}}$. The inset panel presents the superposition of first and last (after $27 \mathrm{~h}$ ) recorded $M^{\prime \prime}$ spectra.

in which $A, \beta$, and $\tau_{\sigma, \infty}$ are constants. This equation describes the $\tau_{\sigma}(t)$ data well (Figure 4), with the fit parameters given in Table 1. It is worth noting that a similar equation has been previously used by Lunkenheimer et al. ${ }^{29}$ for analysis of timedependent dielectric loss data of various glass formers below the glass temperature.

The $\tau_{\text {age }}(T)$ from eq 1 are plotted in Figure 2, where consistency with extrapolation of the $\alpha$-relaxation times from calorimetry is observed. This result supports our premise that the aging rate is governed by the slow structural relaxation dynamics of the glass, so that $\tau_{\text {age }}$ is a measure of $\tau_{\alpha}$ in the glassy state.

The selective narrowing on the high-frequency side of the peaks during aging is illustrated in Figure 4, showing the 


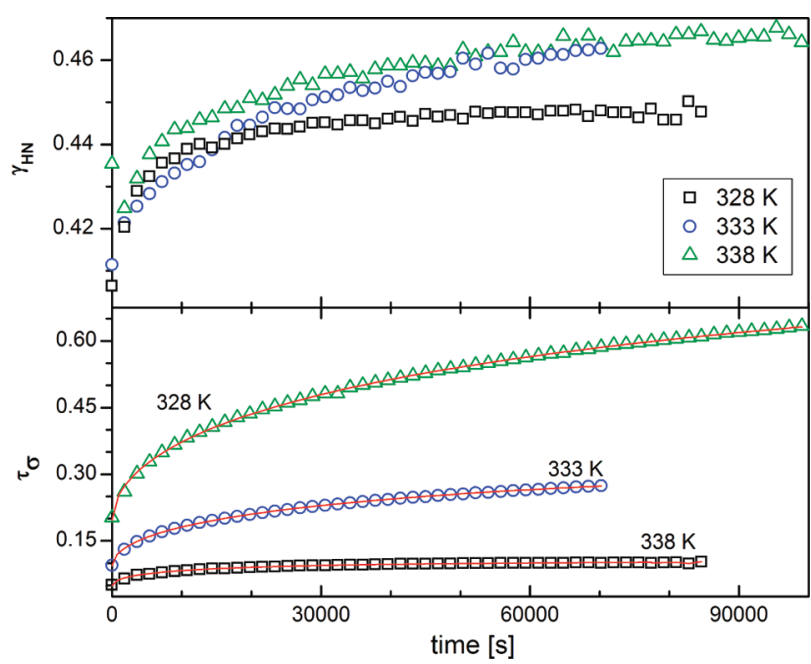

Figure 4. Change during physical aging at the indicated temperatures in (lower) the conductivity relaxation time, along with the fits of eq 1 , and (upper) the shape parameter from eq 2.

Table 1. Fitting Parameters of Equation 1 to the $\tau_{\sigma}(t)$ Experimental Data ${ }^{a}$

\begin{tabular}{cccc}
$T(\mathrm{~K})$ & $A(\mathrm{~s})$ & $\log \left(\tau_{\text {age }} / \mathrm{s}\right)$ & \multicolumn{1}{c}{$\tau_{\sigma, \infty}(\mathrm{s})$} \\
328 & $-0.69 \pm 0.01$ & $5.01 \pm 0.02$ & $0.89 \pm 0.01$ \\
333 & $-0.25 \pm 0.001$ & $4.63 \pm 0.02$ & $0.34 \pm 0.005$ \\
338 & $-0.056 \pm 0.001$ & $4.08 \pm 0.02$ & $0.10 \pm 3.005$
\end{tabular}

${ }^{a}$ The exponent $\beta$ was a temperature-independent 0.53 .

asymmetry shape parameter, $\gamma_{\mathrm{HN}}$, from the Havriliak-Negami function, which for the dielectric modulus is given by

$$
M^{*}=\frac{1}{\varepsilon^{*}}=\left[\varepsilon_{\infty}+\frac{\Delta \varepsilon}{\left(1+(\mathrm{i} \varpi \tau)^{\alpha_{\mathrm{NH}}}\right)^{\gamma_{\mathrm{HN}}}}\right]^{-1}
$$

where $\Delta \varepsilon$, the dielectric strength, $\varepsilon_{\infty}$, and the two exponents are constants. As $\gamma_{\mathrm{HN}}$ goes to unity, there is less skewing toward higher frequencies, the peak becoming more symmetric. This change in the shape of the dispersion is a reflection of the heterogeneous dynamics, potentially arising from two effects: To the extent that ion motion is governed by the size and distribution of vacancies, the densification occasioned by physical aging is presumably more efficient for the more open regions and thus would naturally affect the faster moving ions in such locales. An additional effect, based on the idea of a distribution of relaxation times in a dynamically heterogeneous system, is that each species ages with a time constant identical to its particular relaxation time value. ${ }^{23}$ Thus, species having smaller $\tau_{\alpha}$ contribute at higher frequencies and age more rapidly. These two mechanisms are not unrelated, with the former comprising a specific mechanism for the latter. The consequence is less heterogeneous dynamics, seen in the narrower and more symmetric conductivity relaxation peaks in Figure 3.

To corroborate our interpretation of $\tau_{\text {age }}$ as the time scale for structural relaxation in the glassy state, we apply the same analysis to procaine $\mathrm{HCl}$, another protic ionic liquid. In the inset of Figure 5, the evolution over time of the conductivity relaxation process is displayed.

Additionally, in the main panel of this plot, $\tau_{\sigma}$ for procaine $\mathrm{HCl}$ is plotted versus the aging time at $T=293 \mathrm{~K}\left(T_{\mathrm{g}}=316 \mathrm{~K}\right)$, along with the fit to eq 1 ; the result is $\tau_{\text {age }}=7.59 \times 10^{3} \mathrm{~s}$. Also

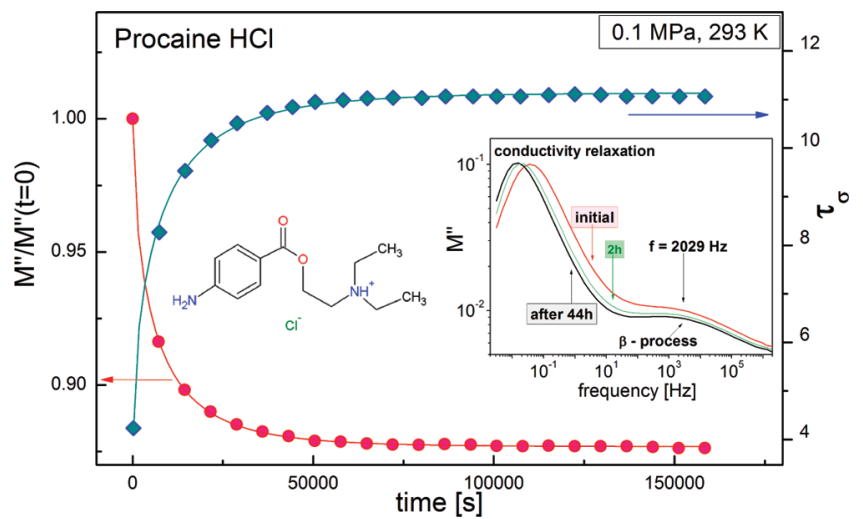

Figure 5. Changes of $\tau_{\sigma}(t)$ (filled diamonds) and $M^{\prime \prime} / M_{(t=0)}^{\prime \prime}(t)$ (filled circles), measured at $293 \mathrm{~K}$ for procaine $\mathrm{HCl}$ (chemical structure depicted). Solid lines are fits of eq 1 (blue line) and the equation of ref 3 (red line) to the experimental data. The evolution in time of the conductivity relaxation process measured at $T=293 \mathrm{~K}$ is shown in the inset.

plotted in the figure is the intensity of the secondary relaxation peak at $2.03 \mathrm{kHz}$, measured at the same temperature. The reduction in the secondary $(\beta)$ relaxation strength as the material physically ages reflects the densification caused by structural relaxation. Thus, as shown in several recent publications, ${ }^{3,30-32}$ the time constant characterizing the change in amplitude of the $\beta$-peak, $\tau_{\beta \text {,age }}$ is essentially the structural relaxation time. The values at $293 \mathrm{~K}$ from the two methods for procaine $\mathrm{HCl}, \tau_{\text {age }}=7.6 \times 10^{3} \mathrm{~s}$ and $\tau_{\text {age }, \beta}=6.0 \times 10^{3} \mathrm{~s}$, differ by about $20 \%$; this near equivalence verifies the analysis introduced in the present work.

In summary, we used the kinetics of the change in the ion conductivity in glassy carvedilol phosphate to probe its structural relaxation. The reliability of our approach is confirmed by agreement between structural relaxation times for procaine hydrochloride determined in this manner with results obtained from analysis of the secondary relaxation. The stability of pharmaceuticals depends on the mobility in the glassy state, and the present method offers a facile means to quantify the dynamics and thereby predict stability.

\section{AUTHOR INFORMATION}

\section{Notes}

The authors declare no competing financial interest.

\section{ACKNOWLEDGMENTS}

The work at NRL was supported by the Office of Naval Research. The support of the Polish State of Committee for Scientific Research (Grant No. N N202 023440) is gratefully acknowledged.

\section{REFERENCES}

(1) Dhinojwala, A.; Wong, G. K.; Torkelson, J. M. Rotational Reorientation Dynamics of Disperse Red 1 in Polystyrene: $\alpha$ Relaxation Dynamics Probed by Second Harmonic Generation and Dielectric Relaxation. J. Chem. Phys. 1994, 100, 6046.

(2) Wagner, H.; Richert, R. Spatial Uniformity of the $\beta$-relaxation in D-Sorbitol. J. Non-Cryst. Solids 1998, 242, 19.

(3) Capaccioli, S.; Prevosto, D.; Lucchesi, M.; Rolla, P. A.; Casalini, R.; Ngai, K. L. Identifying the Genuine Johari-Goldstein $\beta$-Relaxation by Cooling, Compressing, And Aging Small Molecular Glass-Formers. J. Non-Cryst. Solids 2005, 351, 2643. 
(4) Prevosto, D.; Capaccioli, S.; Lucchesi, M.; Rolla, P. A.; Ngai, K. L. Dynamics of Supercooled and Glassy Dipropyleneglycol Dibenzoate As Functions of Temperature and Aging: Interpretation within the Coupling Model Framework. J. Chem. Phys. 2004, 120, 4808.

(5) Casalini, R; Roland, C. M. Aging of the Secondary Relaxation to Probe Structural Relaxation in the Glassy State. Phys. Rev. Lett. 2009, 102, 035701.

(6) Prevosto, D.; Capaccioli, S.; Lucchesi, M.; Rolla, P. A.; Ngai, K. L. Does the Entropy and Volume Dependence of the Structural ARelaxation Originate from the Johari-Goldstein $\beta$-Relaxation? J. NonCryst. Solids 2009, 355, 705.

(7) Wojnarowska, Z.; Paluch, M.; Wlodarczyk, P.; Hawelek, L.; Wrzalik, R.; Zioło, J.; Wygledowska-Kania, M.; Bergler-Czop, B.; Brzezinska-Wcislo, L.; Bujak, P. In Ssearch of Nanoscale Domains with Nematic Order in Supercooled Vitamin A Acetate - Molecular Dynamics Studies. Phys. Rev. E 2011, 83, 051502.

(8) Wojnarowska, Z.; Grzybowska, K.; Hawelek, L.; Swiety-Pospiech, A.; Masiewicz, E.; Paluch, M.; Sawicki, W.; Chmielewska, A.; Bujak, P.; Markowski, J. Molecular Dynamics Studies on the Water Mixtures of Pharmaceutically Important Ionic Liquid Lidocaine HCl. Mol. Pharmaceutics 2012, DOI: $10.1021 / \mathrm{mp} 2005609$.

(9) Wlodarczyk, P.; Paluch, M; Wojnarowska, Z; Hawelek, L; Kaminski, K; Pilch, J. Theoretical and Experimental Studies on the Internal Mobility of Two Sulfonylurea Agents: Glibenclamide and Glimepiride. J. Phys.: Condens. Matter 2011, 23, 425901.

(10) Wojnarowska, Z.; Adrjanowicz, K.; Kaminski, K.; Hawelek, L.; Paluch, M. Effect of Pressure on Tautomers Equilibrium in Supercooled Glibenclamide Drug - Analysis of Fragility Behavior. J. Phys. Chem. B 2010, 114, 14815-14820.

(11) Wojnarowska, Z.; Grzybowska, K.; Adrjanowicz, K.; Kaminski, K.; Paluch, M.; Hawelek, L.; Wrzalik, R.; Dulski, M.; Sawicki, W.; Mazgalski, J.; Tukalska, A.; Bieg, T. Study of the Amorphous Glibenclamide Drug: Analysis of the Molecular Dynamics of Quenched and Cryomilled Material. Mol. Pharm. 2010, 7, 1692-1707.

(12) Wojnarowska, Z.; Adrjanowicz, K.; Wlodarczyk, P.; Kaminska, E.; Kaminski, K.; Grzybowska, K.; Wrzalik, R.; Paluch, M.; Ngai, K. L. Broadband Dielectric Relaxation Study at Ambient and Elevated Pressure of Molecular Dynamics of Pharmaceutical: Indomethacin. J Phys Chem B 2009, 113, 12536.

(13) Adrjanowicz, K.; Wojnarowska, Z.; Wlodarczyk, P.; Kaminski, K.; Paluch, M.; Mazgalski, J. Molecular Mobility in Liquid and Glassy States of Telmisartan (TEL) Studied by Broadband Dielectric Spectroscopy. Eur. J. Pharm. Sci. 2009, 38, 395.

(14) Wojnarowska, Z.; Paluch, M.; Grzybowski, A.; Adrjanowicz, K.; Grzybowska, K.; Kaminski, K.; Wlodarczyk, P.; Pionteck, J. Study of Molecular Dynamics of Pharmaceutically Important Protic Ionic Liquid-Verapamil Hydrochloride I. Test of Thermodynamic Scaling. J. Chem. Phys. 2009, 131, 104505.

(15) Adrjanowicz, K.; Kaminski, K.; Wojnarowska, Z.; Dulski, M.; Hawelek, L.; Pawlus, S.; Paluch, M. Dielectric Relaxation and Crystallization Kinetics of Ibuprofen at Ambient and Elevated Pressure. J. Phys. Chem. B 2010, 114, 6579-6593.

(16) Wojnarowska, Z.; Hawelek, L.; Paluch, M.; Sawicki, W.; Ngai, K. L. Molecular Dynamics at Ambient and Elevated Pressure of the Amorphous Pharmaceutical: Nonivamide (Pelargonic Acid Vanillylamide). J. Chem. Phys. 2011, 28, 044517.

(17) Richert, R.; Weinstein, S. Nonlinear Dielectric Response and Thermodynamic Heterogeneity in Liquids. Phys. Rev. Lett. 2006, 97, 095703.

(18) Wang, L. M.; Richert, R. Measuring the Configurational Heat Capacity of Liquids. Phys. Rev. Lett. 2007, 99, 185701.

(19) Huang, W.; Richert, R. Dynamics of Glass-Forming Liquids. XIII. Microwave Heating in Slow Motion. J. Chem. Phys. 2009, 130, 194509.

(20) Rzoska, S. J.; Drozd-Rzoska, A. Dual Field Nonlinear Dielectric Spectroscopy in a Glass Forming EPON 828 Epoxy Resin. J. Phys.: Condens. Matter 2012, 24, 035101.
(21) Schiener, B.; Bohmer, R.; Loidl, A.; Chamberlin, R. V. Nonresonant Spectral Hole Burning in the Slow Dielectric Response of Supercooled Liquids. Science 1996, 274, 752.

(22) Roland, C. M.; Fragiadakis, D.; Coslovich, D.; Capaccioli, S.; Ngai, K. L. Correlation of Nonexponentiality with Dynamic Heterogeneity from Four-Point Dynamic Susceptibility $\chi_{4}(t)$ and Its Approximation $\chi_{T}(t) . J$. Chem. Phys. 2010, 133, 124507.

(23) Richert, R. Physical Aging and Heterogeneous Dynamics. Phys. Rev. Lett. 2010, 104, 085702.

(24) Angell, C. A.; Byrne, N.; Belieres, J. P. Parallel Developments in Aprotic and Protic Ionic Liquids: Physical Chemistry and Applications. Acc. Chem. Res. 2007, 40, 1228.

(25) Greaves, T. L.; Weerawardena, A.; Fong, C.; Krodkiewska, I.; Drummond, C. J. Protic Ionic Liquids: Solvents with Tunable Phase Behavior and Physicochemical Properties. J. Phys. Chem. B 2006, 110, 22479.

(26) Wojnarowska, Z.; Roland, C. M.; Swiety-Pospiech, A.; Grzybowska, K.; Paluch, M. Anomalous Electrical Conductivity Behavior at Elevated Pressure in the Protic Ionic Liquid Procainamide Hydrochloride. Phys. Rev. Lett. 2012, 108, 015701.

(27) Pimenov, A.; Lunkenheimer, P.; Rall, H.; Kohlhaas, R.; Loidl, A.; Bohmer, R. Ion Transport in the Fragile Glass Former $3 \mathrm{KNO}_{3}-$ $2 \mathrm{Ca}\left(\mathrm{NO}_{3}\right)_{2}$. Phys. Rev. E 1996, 54, 676.

(28) Wagner, H.; Richert, R. Measurement and Analysis of TimeDomain Electric Field Relaxation: The Vitreous Ionic Conductor $0.4 \mathrm{Ca}\left(\mathrm{NO}_{3}\right)_{2}-0.6 \mathrm{KNO}_{3}$. J. Appl. Phys. 1999, 85, 1750.

(29) Lunkenheimer, P.; Wehn, R.; Schneider, U.; Loidl, A. Glassy Aging Dynamics. Phys. Rev. Lett. 2005, 95, 055702.

(30) Boucher, V. M.; Cangialosi, D.; Alegría, A.; Colmenero, J. Accelerated Physical Aging in PMMA/Silica Nanocomposites. Soft Matter 2010, 6, 3306.

(31) Kaminski, K.; Adrjanowicz, K.; Kaminska, E.; Paluch, M. Probing of Structural Relaxation Times in the Glassy State of Sucrose and Trehalose Based on Dynamical Properties of Two Secondary Relaxation Processes. Phys. Rev. E 2011, 83, 061502.

(32) Casalini, R.; Roland, C. M. Aging of a Low Molecular Weight Poly(methyl methacrylate). J. Non-Cryst. Solids 2011, 357, 282. 